\title{
Light meson spectroscopy at BESIII
}

\author{
Tianjue $\operatorname{Min}^{1, \star}$ \\ for the BESIII Collaboration \\ ${ }^{1}$ Institute of High Energy Physics, Beijing 100049, People's Republic of China
}

\begin{abstract}
The BESIII collaboration has collected a sample of 1.311 billion $J / \psi$ events since 2009. In this talk, we will introduce four recent analyses on light meson spectroscopy at BESIII, including two studies on the $X(1835)$ and two partial wave analyses that are related to searching for and studying glueball candidates.
\end{abstract}

\section{Introduction}

Quantum Chromodynamics is one of the fundamental theories in modern high energy physics. Light meson spectroscopy plays a crucial role in examining and understanding the QCD theory in nonpertubative energy region. Decays of the $J / \psi$ meson, being the lowest lying $1^{--} c \bar{c}$ states, provide an ideal laboratory for light meson spectroscopy.

The BESIII detector collected 225 million $J / \psi$ events in 2009 and 1.086 billion $J / \psi$ events in 2012, which gives us a very good opportunity to study light mesons through $J / \psi$ decays. In this presentation, we will introduce some recent results from BESIII that are related to light meson spectroscopy.

\section{Recent results from BESIII}

\subsection{Observation of $X(1835)$ in $J / \psi \rightarrow \gamma K_{S}^{0} K_{S}^{0} \eta$}

The state $X(1835)$ was first observed by the BESII experiment as a peak in the $\eta^{\prime} \pi^{+} \pi^{-}$invariant mass distribution in $J / \psi \rightarrow \gamma \eta^{\prime} \pi^{+} \pi^{-}$decays [1]. This observation was later confirmed by BESIII studies

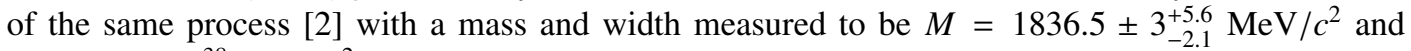
$\Gamma=190 \pm 9_{-36}^{+38} \mathrm{MeV} / c^{2}$. An anomalously strong enhancement at the proton-antiproton $(p \bar{p})$ mass threshold, dubbed $X(p \bar{p})$, was first observed by BESII in $J / \psi \rightarrow \gamma p \bar{p}$ decays [3]; this observation was confirmed by BESIII [4] and CLEO [5]. This enhancement structure was subsequently determined to have spin-parity $J^{P}=0^{-}$by BESIII [6]. Among the various theoretical interpretations on the nature of the $X(1835)$ and $X(p \bar{p})$, a particularly intriguing one suggests that the two structures originate from a $p \bar{p}$ bound state [7-10].

To understand the nature of the $X(1835)$, it is crucial to measure its $J^{P C}$ and to search for new decay modes. Recently, BESIII analyzed the $J / \psi \rightarrow \gamma K_{S}^{0} K_{S}^{0} \eta$ process with 1.311 billion $J / \psi$ data sample collected since 2009 [11]. The scatter plot of the invariant mass of $K_{S}^{0} K_{S}^{0}$ versus that of $K_{S}^{0} K_{S}^{0} \eta$ is shown in Fig. 1(a). A clear accumulation of events is seen around the intersection of the

^e-mail: mintj@ihep.ac.cn 
$f_{0}(980)$ on $M_{K_{S}^{0}} K_{S}^{0}$ and the structure around $1.85 \mathrm{GeV} / c^{2}$ on $M_{K_{S}^{0}} K_{S}^{0} \eta$. This indicates that the structure around $1.85 \mathrm{GeV} / c^{2}$ is strongly correlated with the $f_{0}(980)$. A partial wave analysis (PWA) of events satisfying $M_{K_{S}^{0} K_{S}^{0} \eta}<2.8 \mathrm{GeV} / c^{2}$ and $M_{K_{S}^{0} K_{S}^{0}}<1.1 \mathrm{GeV} / c^{2}$ is performed to determine the parameters of the structure around $1.85 \mathrm{GeV} / c^{2}$. The $K_{S}^{0} K_{S}^{0} \eta$ and $K_{S}^{0} K_{S}^{0}$ mass spectra are shown in Fig. 1(b) and (c). The PWA fit requires a contribution from $X(1835) \rightarrow K_{S}^{0} K_{S}^{0} \eta$ with a statistical significance greater than $12.9 \sigma$, where the $X(1835) \rightarrow K_{S}^{0} K_{S}^{0} \eta$ is dominated by $f_{0}(980)$ production. The spin parity of the $X(1835)$ is determined to be $0^{-+}$, which is significantly better than the $1^{++}$or $2^{-+}$hypotheses. The mass and width of the $X(1835)$ are measured to be $1844 \pm 9$ (stat $)_{-25}^{+16}$ (syst) $\mathrm{MeV} / c^{2}$ and $192_{-17}^{+20}$ (stat) ${ }_{-43}^{+62}$ (syst) $\mathrm{MeV} / c^{2}$, respectively. The corresponding product branching fraction is measured to be $\left(3.31_{-0.30}^{+0.33}(\mathrm{stat})_{-1.29}^{+1.96}(\mathrm{syst})\right) \times 10^{-5}$. The mass and width of the $X(1835)$ are consistent with the values obtained from the decay $J / \psi \rightarrow \gamma \eta^{\prime} \pi^{+} \pi^{-}$by BESIII [1]. Another $0^{-+}$state, the $X(1560)$, also is observed in data with a statistical significance larger than $8.9 \sigma$ and is seen to interfere with the $X(1835)$. The mass and width of the $X(1560)$ are consistent with those of the $\eta(1405)$ and $\eta(1475)$ as given in [12] within $2.0 \sigma$ and $1.4 \sigma$, respectively. Present statistics do not allow us to conclusively determine if the $X(1560)$ is the same state as the $\eta(1405) / \eta(1475)$ or a new meson.
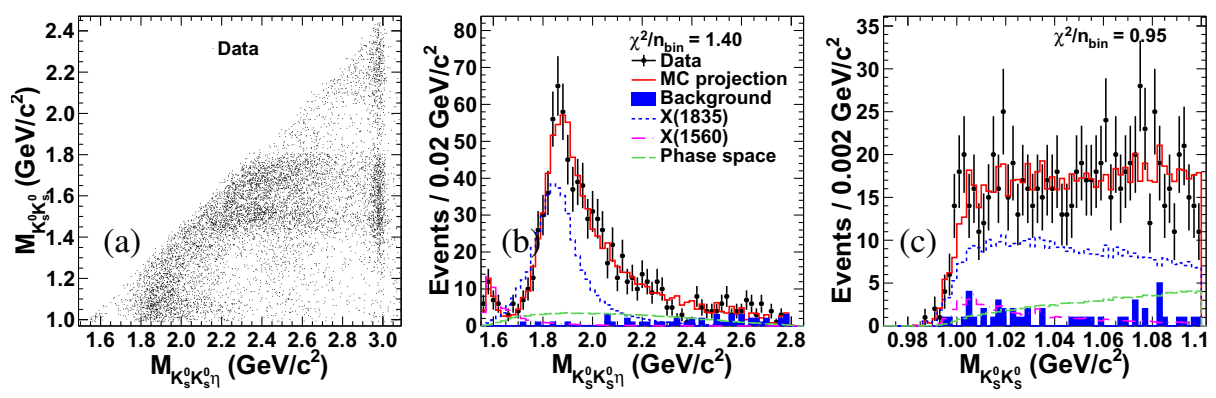

Figure 1. (a) Scatter plot of $M_{K_{S}^{0} K_{S}^{0}}$ versus that of $M_{K_{S}^{0} K_{S}^{0} \eta}$; (b) and (c) are the comparisons of invariant mass distributions of $K_{S}^{0} K_{S}^{0} \eta$ and $K_{S}^{0} K_{S}^{0}$ between data and PWA fit projections.

\subsection{Anomalous line shape of $\eta^{\prime} \pi^{+} \pi^{-}$near the $p \bar{p}$ mass threshold in $J / \psi \rightarrow \gamma \eta^{\prime} \pi^{+} \pi^{-}$}

From Sect. 2.1 we know that the $X(1835)$ has the same $J^{P C}$ number as the $X(p \bar{p})$ does. If the $X(1835)$ is really a $p \bar{p}$ bound state, it should have a strong coupling to $0^{-} p \bar{p}$ systems, in which case the line shape of the $X(1835)$ at the $p \bar{p}$ mass threshold would be affected by the opening of the $X(1835) \rightarrow p \bar{p}$ decay mode. A study of the $\eta^{\prime} \pi^{+} \pi^{-}$line shape of $X(1835)$ with high statistical precision therefore provides valuable information that helps clarify the nature of the $X(1835)$ and $X(p \bar{p})$.

With 1.086 billion $J / \psi$ data collected in 2012, BESIII re-analysed the $J / \psi \rightarrow \gamma \eta^{\prime} \pi^{+} \pi^{-}$process [13] in which the $X(1835)$ was observed $[1,2]$. The $\eta^{\prime}$ is reconstructed in its two major decay modes: $\eta^{\prime} \rightarrow \gamma \pi^{+} \pi^{-}$and $\eta^{\prime} \rightarrow \eta \pi^{+} \pi^{-}, \eta \rightarrow \gamma \gamma$. As shown in Fig. 2, we observed a significant abrupt change in slope of the $X(1835) \rightarrow \eta^{\prime} \pi^{+} \pi^{-}$line shape at the $p \bar{p}$ mass threshold. Study of background shows this abrupt change in line shape is not caused by background processes.

A simultaneous fit to the $\eta^{\prime} \pi^{+} \pi^{-}$mass distribution between 1.3 and $2.25 \mathrm{GeV} / c^{2}$ for both event samples is performed. We find that a simple Breit-Wigner function cannot describe the $X(1835)$ line shape near the $p \bar{p}$ mass threshold (Fig. 3(a)). Typically, there are two circumstances where an abrupt distortion of a resonance's line shape shows up: a threshold effect caused by the opening of 

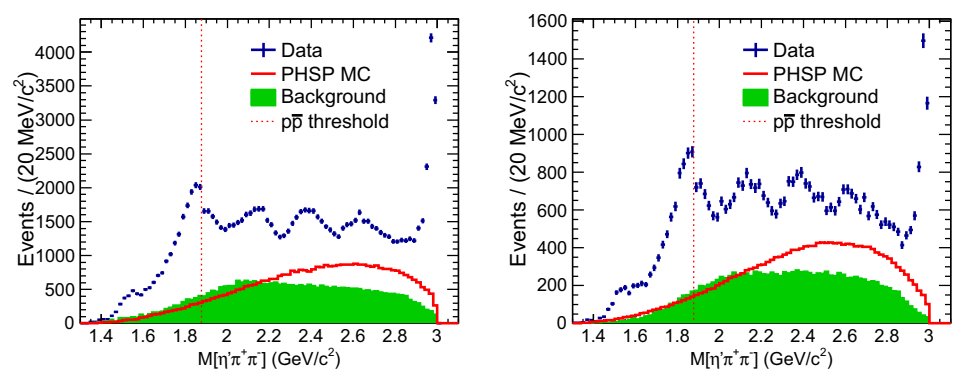

Figure 2. The $\eta^{\prime} \pi^{+} \pi^{-}$invariant mass spectra after the application of all selection criteria. The plot on the left side shows the spectrum for events with the $\eta^{\prime} \rightarrow \gamma \pi^{+} \pi^{-}$channel, and that on the right shows the spectrum for the $\eta^{\prime} \rightarrow \eta \pi^{+} \pi^{-}, \eta \rightarrow \gamma \gamma$ channel.

an additional decay mode, or interference between two resonances. We try to fit the data for both of these possibilities.

In the first model, we assume the state around $1.85 \mathrm{GeV} / c^{2}$ couples to the $p \bar{p}$ and use a Flatte formula [14] to describe the $X(1835)$ line shape:

$$
T=\frac{\sqrt{\rho_{\text {out }}}}{\mathcal{M}^{2}-s-i \sum_{k} g_{k}^{2} \rho_{k}} \approx \frac{\sqrt{\rho_{\text {out }}}}{\mathcal{M}^{2}-s-i g_{0}^{2}\left(\rho_{0}+\frac{g_{p \bar{p}}^{2}}{g_{0}^{2}} \rho_{p \bar{p}}\right)}
$$

Here, $T$ is the decay amplitude, $\sqrt{\rho_{\text {out }}}$ is the phase space for $J / \psi \rightarrow \gamma \eta^{\prime} \pi^{+} \pi^{-}, \mathcal{M}$ is a parameter with the dimension of mass, $s$ is the square of the $\eta^{\prime} \pi^{+} \pi^{-}$system's mass, $\rho_{k}$ is the phase space for decay mode $k$, and $g_{k}^{2}$ is the corresponding coupling strength. The term $\sum_{k} g_{k}^{2} \rho_{k}$ describes how the decay width varies with $s$, and can be replaced by $g_{0}^{2}\left(\rho_{0}+\frac{g_{p \bar{p}}^{2}}{g_{0}^{2}} \rho_{p \bar{p}}\right)$ approximately, where $g_{0}^{2}$ is the sum of $g^{2}$ of all decay modes other than the $X(1835) \rightarrow p \bar{p}, \rho_{0}$ is the maximum two-body decay phase space volume [12], and $g_{p \bar{p}}^{2} / g_{0}^{2}$ is the ratio between the coupling strength to the $p \bar{p}$ channel and the sum of all other channels. The fit results for this model are shown in Fig. 3(b). The Flatté model fit yields $\mathcal{M}=1638.0 \pm 121.9$ (stat) ${ }_{-254.3}^{+127.8}$ (syst) $\mathrm{MeV} / c^{2}, g_{0}^{2}=93.7 \pm 35.4$ (stat) ${ }_{-43.9}^{+47.6}(\mathrm{syst})\left(\mathrm{GeV} / c^{2}\right)^{2}, g_{p \bar{p}}^{2} / g_{0}^{2}=$ $2.31 \pm 0.37$ (stat) ${ }_{-0.60}^{+0.83}$ (syst), a product branching fraction of $\mathcal{B}(J / \psi \rightarrow \gamma X) \cdot \mathcal{B}\left(X \rightarrow \eta^{\prime} \pi^{+} \pi^{-}\right)=(3.93 \pm$ $0.38(\text { stat })_{-0.84}^{+0.31}$ (syst) $) \times 10^{-4}$. The value of $g_{p \bar{p}}^{2} / g_{0}^{2}$ implies that the couplings between the state around $1.85 \mathrm{GeV} / c^{2}$ and the $p \bar{p}$ final states is very large, the significance of $g_{p \bar{p}}^{2} / g_{0}^{2}$ being non-zero is larger than $7 \sigma$. Following the definitions given in [15], the pole nearest to the $p \bar{p}$ mass threshold is found to be $M_{\text {pole }}=1909.5 \pm 15.9$ (stat $)_{-27.5}^{+9.4}$ (syst) $\mathrm{MeV} / c^{2}$ and $\Gamma_{\text {pole }}=273.5 \pm 21.4(\text { stat })_{-64.0}^{+6.1}($ syst $) \mathrm{MeV} / c^{2}$. In this fit, an additional Breit-Wigner resonance (denoted as " $X(1920)$ " in Fig. 3(b)) is needed with 5.7 $\sigma$ statistical significance.

In the second model, we assume the existence of a narrow resonance near the $p \bar{p}$ threshold and that the interference between this resonance and the $X(1835)$ produces the line shape distortion. Here we denote this narrow resonance as " $X(1870)$." For this case we represent the line shape in the vicinity on $1835 \mathrm{MeV} / c^{2}$ by the square of $T$, which is the coherent sum of two Breit-Wigner amplitudes. The fit results for this model are shown in Fig. 3(c). The $X(1835)$ mass is $1825.3 \pm$ 2.4 (stat) $+17.3_{-2.4}$ (syst) $\mathrm{MeV} / c^{2}$ and width is $245.2 \pm 13.1$ (stat) ${ }_{-9.6}^{+4.6}$ (syst) $\mathrm{MeV} / c^{2}$; The $X(1870$ ) mass is $1870.2 \pm 2.2$ (stat) ${ }_{-0.7}^{+2.3}$ (syst) $\mathrm{MeV} / c^{2}$ and width is $13.0 \pm 6.1$ (stat) ${ }_{-3.8}^{+2.1}$ (syst) $\mathrm{MeV} / c^{2}$, with a statistical significance that is larger than $7 \sigma$. In the parameterization of this model, there exist two solutions 

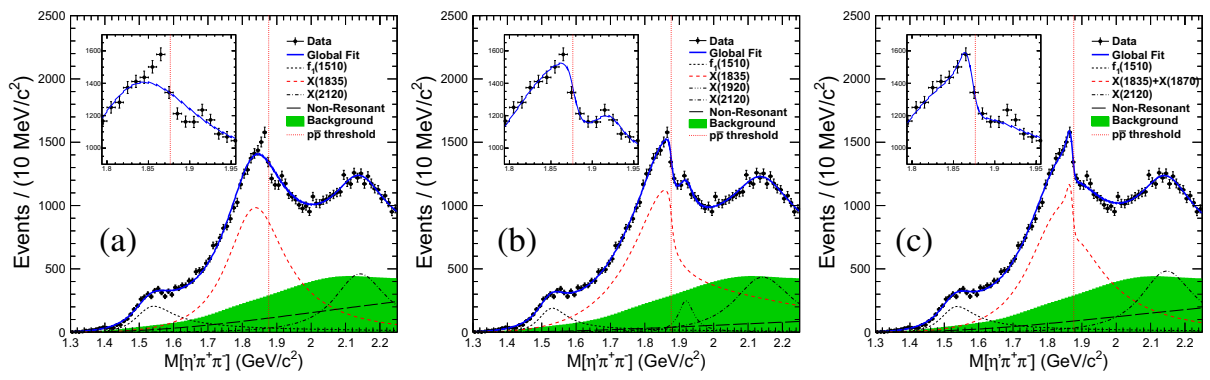

Figure 3. Fit results of using (a) simple Breit-Wigner formula; (b) the Flatté formula; (c) coherent sum of two Breit-Wigner amplitudes.

which only differ in branching fractions of the two Breit-Wigner functions with each other [16]. The product branching fractions with constructive interference are $\mathcal{B}[J / \psi \rightarrow \gamma X(1835)] \cdot \mathcal{B}[X(1835) \rightarrow$ $\left.\left.\eta^{\prime} \pi^{+} \pi^{-}\right]=(3.01 \pm 0.17 \text { (stat })_{-0.28}^{+0.26}(\mathrm{syst})\right) \times 10^{-4}$ and $\mathcal{B}[J / \psi \rightarrow \gamma X(1870)] \cdot \mathcal{B}\left[X(1870) \rightarrow \eta^{\prime} \pi^{+} \pi^{-}\right]=$ $\left(2.03 \pm 0.12\right.$ (stat) ${ }_{-0.35}^{+0.18}($ syst $\left.)\right) \times 10^{-7}$, while the solution with destructive interference gives $\mathcal{B}[\mathrm{J} / \psi \rightarrow$ $\gamma X(1835)] \cdot \mathcal{B}\left[X(1835) \rightarrow \eta^{\prime} \pi^{+} \pi^{-}\right]=\left(3.72 \pm 0.21(\text { stat })_{-0.70}^{+0.43}(\mathrm{syst})\right) \times 10^{-4}$, and $\mathcal{B}[J / \psi \rightarrow$ $\left.\gamma X(1870)] \cdot \mathcal{B}\left[X(1870) \rightarrow \eta^{\prime} \pi^{+} \pi^{-}\right]=(1.57 \pm 0.09 \text { (stat })_{-0.86}^{+0.49}(\mathrm{syst})\right) \times 10^{-5}$. In this model, the $X(1920)$ is not included in the fit because its significance is just $3.9 \sigma$.

With current data, both models fit the data well with fit qualities, more sophisticated models such as a mixture of above two models cannot be ruled out. But both fits do suggest the existence of either a $p \bar{p}$ molecule-like or bound state.

\subsection{Model independent partial wave analysis of $J / \psi \rightarrow \gamma \pi^{0} \pi^{0}$}

The $J / \psi$ radiative decays to two pesudoscalars are very important channels for identifying scalar and tensor glueballs. Recently, BESIII studied the $J / \psi \rightarrow \gamma \pi^{0} \pi^{0}$ process with model independent partial wave analysis [17]. In the PWA result, two types of ambiguities are present. Trivial ambiguities arise due to the possibility of the overall amplitude in each bin to be rotated by $\pi$ or to be reflected over the real axis in the complex plane. These may be partially addressed by applying a phase convention to the results of the fits. Non-trivial ambiguities arise from the freedom of amplitudes with the same quantum numbers to have different phases. The non-trivial ambiguities represent a greater challenge to the analysis and cannot be eliminated without introducing model dependencies. The intensity for each amplitude as a function of $M_{\pi^{0} \pi^{0}}$ is shown in Fig. 4. For $2^{++}$contributions, the phase differences with respect to the reference amplitude $\left(2^{++} E 1\right)$, is constrained to be real. Above the $K \bar{K}$ threshold, two distinct sets of solutions are apparent in most bins as expected. These results may be combined with those of similar reactions for a more comprehensive study of the light scalar meson spectrum. The branching fraction of radiative $J / \psi$ decays to $\pi^{0} \pi^{0}$ is measured to be $(1.15 \pm 0.05) \times 10^{-3}$, where the error is systematic only and the statistical error is negligible. This is the first measurement of this branching fraction.

\subsection{Partial wave analysis of $J / \psi \rightarrow \gamma \phi \phi$}

The mass of the lowest lying pseudoscalar glueball is predicted to be around $2.3-2.6 \mathrm{GeV} / c^{2}$ by Lattice QCD [18-20]. Aside from the $\eta(2225)$, very little is known in the pseudoscalar sector above 

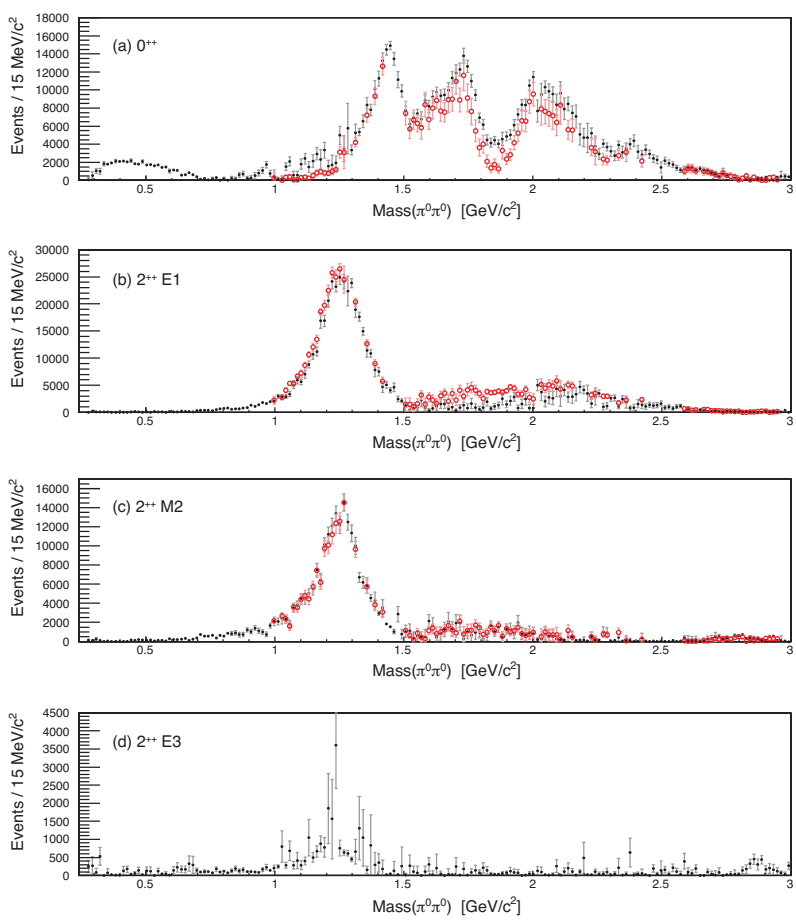

Figure 4. The intensities for the (a) $0^{++}$, (b) $2^{++} \mathrm{E} 1$, (c) $2^{++} \mathrm{M} 2$ and (d) $2^{++} \mathrm{E} 3$ amplitudes. The solid black markers show the phase differences calculated from one set of solutions, while the open red markers represent the ambiguous partner solutions.

$2 \mathrm{GeV} / c^{2}$. The $J / \psi$ radiative decays to two vector particles processes provide opportunities to study pseudoscalar particles. Recently, BESIII studied the $J / \psi \rightarrow \gamma \phi \phi$ process with a PWA [21]. The results are shown in Fig. 5. $0^{-+}$states are dominant components in the PWA solution. The existence of the $\eta(2225)$ is confirmed and two additional pseudoscalar states, $\eta(2100)$ with a mass of $230_{-35}^{+64}$ (stat) ${ }_{-26}^{+77}$ (syst) $\mathrm{MeV} / c^{2}$ and a width of $250_{-30}^{+36}$ (stat) ${ }_{-164}^{+187}$ (syst) $\mathrm{MeV} / c^{2}$, and $X(2500)$ with a mass of $2470_{-19}^{+15}$ (stat) ${ }_{-23}^{+63}$ (syst) $\mathrm{MeV} / \mathrm{c}^{2}$ and a width of $230_{-35}^{+64}$ (stat) ${ }_{-33}^{+53}$ (syst) $\mathrm{MeV} / c^{2}$, are obtained. The new experimental results are helpful for mapping out pseudoscalar excitations and searching for a $0^{-+}$ glueball. The three tensors $f_{2}(2100), f_{2}(2300)$, and $f_{2}(2340)$ which were observed in $\pi N$ scattering are also observed in $J / \psi \rightarrow \gamma \phi \phi$.

\section{Summary}

With the largest $J / \psi$ data sample in the world, the BESIII collaboration has significant contributions in light meson spectroscopy. For the first time, the $X(1835)$ is determined to be a $0^{-+}$state; an anomalous line shape of $X(1835) \rightarrow \eta^{\prime} \pi^{+} \pi^{-}$at the $p \bar{p}$ mass threshold is observed, which suggests the existence of a $p \bar{p}$ molecule like or bound state; sophisticated model independent PWA of $J / \psi \rightarrow \gamma \pi^{0} \pi^{0}$ improves our understanding of the rich structures in the $\pi \pi$ system and provides valuable comparison with the $\eta \eta$ [22] and $K K$ (both model dependent and independent PWA are ongoing at BESIII) systems; the $\phi \phi$ system has been investigated to search for pseudoscalar and tensor glueballs. With the high statistics data accumulated at the BESIII detector, more interesting results are expected in the future. 

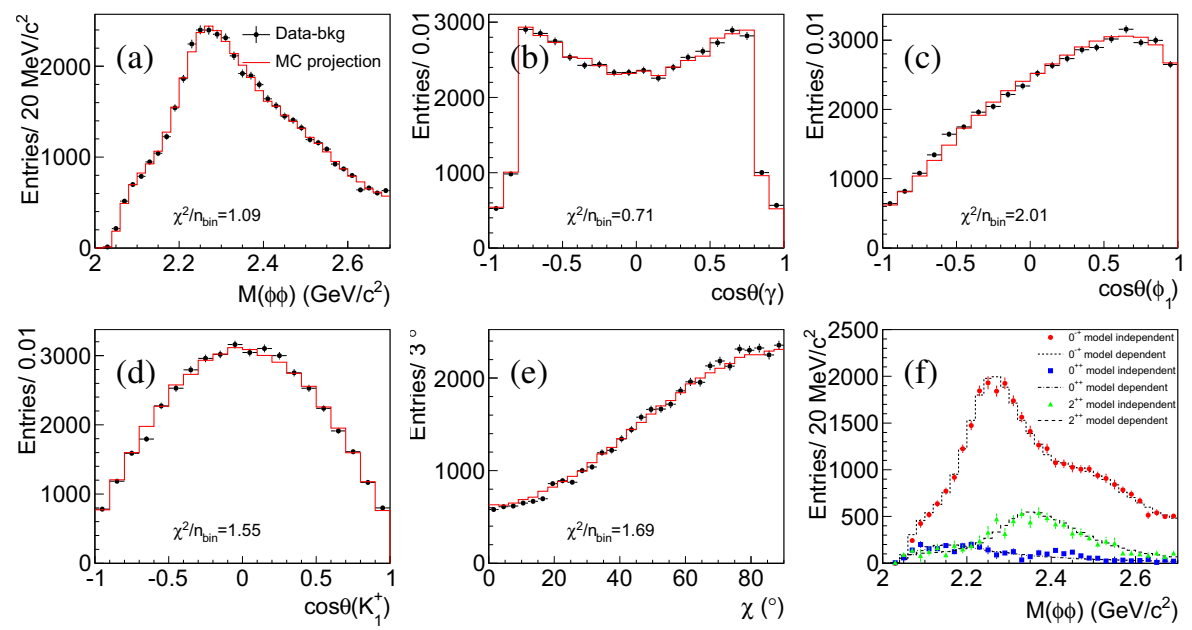

Figure 5. Superposition of data and the PWA fit projections for: (a) invariant mass distributions of $\phi \phi$; (b) $\cos \theta$ of $\gamma$ in the $J / \psi$ rest frame; (c) $\cos \theta$ of $\phi_{1}$ in the $X$ rest frame; (d) $\cos \theta$ of $K^{+}$in the $\phi_{1}$ rest frame; (e) the azimuthal angle between the normals to the two decay planes of $\phi$ in the $X$ rest frame; (f) Intensities of individual $J^{P C}$ components.

\section{References}

[1] M. Ablikim et al. (BES Collaboration), Phys. Rev. Lett. 95, 262001 (2005)

[2] M. Ablikim et al. (BESIII Collaboration), Phys. Rev. Lett. 106, 072002 (2011)

[3] J. Z. Bai et al. (BES Collaboration), Phys. Rev. Lett. 91, 022001 (2003)

[4] M. Ablikim et al. (BESIII Collaboration), Chin. Phys. C 34, 421 (2010)

[5] J. P. Alexander et al. (CLEO Collaboration), Phys. Rev. D 82, 092002 (2010)

[6] M. Ablikim et al. (BESIII Collaboration), Phys. Rev. Lett. 108, 112003 (2012)

[7] S. L. Zhu and C. S. Gao, Commun. Theor. Phys. 46, 291 (2006)

[8] J. P. Dedonder, B. Loiseau, B. El-Bennich, and S. Wycech, Phys. Rev. C 80, 045207 (2009)

[9] C. Liu, Eur. Phys. J. C 53, 413 (2008)

[10] Z. G. Wang and S. L. Wan, J. Phys. G 34, 505 (2007)

[11] M. Ablikim et al. (BESIII Collaboration), Phys. Rev. Lett. 115, 091803 (2015)

[12] K. A. Olive et al. (Particle Data Group), Chin. Phys. C 38, 090001 (2014)

[13] M. Ablikim et al. (BESIII Collaboration), Phys. Rev. Lett. 117, 042002 (2016)

[14] S. M. Flatté, Phys. Lett. B 63, 224 (1976)

[15] B. S. Zou and D. V. Bugg, Phys. Rev. D 48, R3948 (1993)

[16] K. Zhu, X. H. Mo, C. Z. Yuan, and P. Wang, Int. J. Mod. Phys. A 26, 4511 (2011)

[17] M. Ablikim et al. (BESIII Collaboration), Phys. Rev. D 92, 052003 (2015)

[18] G. S. Bali et al. (UKQCD Collaboration), Phys. Lett. B 309, 378 (1993)

[19] C. J. Morningstar and M. Peardon, Phys. Rev. D 60, 034509 (1999)

[20] Y. Chen et al., Phys. Rev. D 73, 014516 (2006)

[21] M. Ablikim et al. (BESIII Collaboration), Phys. Rev. D 93, 112011 (2016)

[22] M. Ablikim et al. (BESIII Collaboration), Phys. Rev. D 87, 092009 (2013) 